\title{
Acute type A aortic dissection extending beyond ascending aorta: Limited or extensive distal repair
}

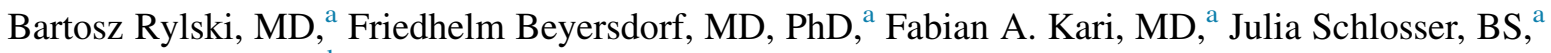 \\ Philipp Blanke, MD, ${ }^{\mathrm{b}}$ and Matthias Siepe, $\mathrm{MD}^{\mathrm{a}}$
}

Objective: The aim of our study was to delineate the effect of aortic arch surgery extension on the outcomes in acute type A dissection extending beyond the ascending aorta.

\begin{abstract}
Methods: From 2001 to 2013, of 197 patients with type A dissection, 153 (78\%) with dissection extending beyond the ascending aorta (age, 61 years; first quartile, 50; third quartile, 69; 67\% men) were identified. Aortic repair involved isolated ascending replacement $(\mathrm{n}=102)$, hemiarch $(\mathrm{n}=37)$, and total arch replacement $(\mathrm{n}=14)$. The median follow-up period was 4.9 years (first quartile, 2.5; third quartile, 7.6; 733 patient-years).
\end{abstract}

Results: In-hospital mortality was $9.8 \%, 21.6 \%$, and $28.6 \%(P=.122)$ for patients with no, hemiarch, and total arch replacement. Age $>80$ years (odds ratio [OR], 9.37; $P=.006$ ), malperfusion syndrome (OR, 4.74; $P=.004)$, and total arch replacement (OR, 6.47; $P=.016)$ were independent predictors of perioperative mortality. Freedom from distal reintervention was $93 \% \pm 3 \%, 97 \% \pm 3 \%$, and $100 \%$ at 1 year and $89 \% \pm 3 \%, 97 \% \pm 3 \%$, and $100 \%$ at 5 years for the no, hemiarch, and total arch replacement groups, respectively (log-rank, $P=.440)$. Marfan syndrome (OR, 12.40; $P=.038)$ and dissection of all aortic segments $(\mathrm{OR}, 10.68 ; P=.007)$ predicted distal aortic reintervention. In-hospital mortality for elective reintervention was $0 \%$.

Conclusions: Limiting the extent of surgery for type A aortic dissection to ascending aortic replacement was associated with low perioperative mortality. Thus, aortic arch repair can be deferred, because it can be performed electively with a lower mortality risk. (J Thorac Cardiovasc Surg 2014;148:949-54)

In the acute setting of Stanford type A aortic dissection, emergency surgical intervention has primarily aimed at preserving life by preventing aortic rupture, correcting aortic valve insufficiency, and restoring flow to the dissected branch vessels. ${ }^{1}$ In patients with dissection confined to the proximal aorta, the entire dissected aortic segment can be repaired by sole ascending aortic replacement. ${ }^{2,3}$ However, in most patients, the dissection process will extend beyond the ascending aorta, ${ }^{4}$ and replacement of the entire dissected tissue is usually not feasible.

Complete resection of the intimal tear and prosthetic replacement of the ascending aorta are considered the standard of care for type A dissection surgery. In patients with aortic arch aneurysm or an intimal tear localized to

\footnotetext{
From the Heart Center Freiburg University, ${ }^{\text {a }}$ Freiburg, Germany; and Department of Diagnostic and Interventional Radiology, ${ }^{\mathrm{b}}$ University Hospital of Würzburg, Würzburg, Germany.

Disclosures: Authors have nothing to disclose with regard to commercial support.

Read at the 94th Annual Meeting of The American Association for Thoracic Surgery, Toronto, Ontario, Canada, April 26-30, 2014.

Received for publication Jan 7, 2014; revisions received March 12, 2014; accepted for publication May 19, 2014; available ahead of print July 10, 2014.

Address for reprints: Bartosz Rylski, MD, Heart Center Freiburg University, Hugstetter Str 55, Freiburg 79106, Germany (E-mail: bartosz.rylski@ universitaets-herzzentrum.de).

$0022-5223 / \$ 36.00$

Copyright (C) 2014 by The American Association for Thoracic Surgery

http://dx.doi.org/10.1016/j.jtcvs.2014.05.051
}

the aortic arch, more extensive surgery with hemi- or total arch replacement is warranted. ${ }^{1}$ However, the surgical strategy for patients with the dissection process extending into the aortic arch but without an arch aneurysm or intimal tear within the arch remains controversial. Considering the still high perioperative mortality for patients with type A dissection of $8 \%$ to $34 \%,{ }^{5-7}$ the increased perioperative risk associated with more extensive distal aortic repair must be weighed against the risk of future reintervention and associated mortality.

In the present study, we investigated the effect of aortic arch surgery extension on early and intermediate outcomes in patients with acute type A dissection, with the dissection process extending beyond the ascending aorta, and analyzed the effect of more extensive aortic arch replacement for prevention of subsequent distal aortic reintervention.

\section{METHODS}

\section{Study Population and Definitions}

From 2001 to 2013, of 197 patients who underwent surgery for acute Stanford type A aortic dissection, 153 (78\%) had presented with dissection extending beyond the ascending aorta and involvement of at least the aortic arch. Patients with the dissection process extending into the aortic arch were further analyzed and constituted the study population. They were divided into groups according to the extension of aortic arch surgery: isolated ascending aortic arch, hemiarch, and total arch replacement. The demographics and clinical characteristics are presented in Table 1. 


\section{Abbreviation and Acronym \\ $\mathrm{OR}=$ odds ratio}

Acute aortic dissection was defined as dissection surgically treated no later than 14 days after symptom onset. Distal reintervention was defined as open or endovascular intervention on the aorta distal to the ascending aortic and/or arch prosthesis implanted by the initial surgery. The institutional review committee approved the present retrospective study, and the need for informed consent was waived.

\section{Surgical Management}

According to our institutional policies, surgery on the aortic arch was routinely performed under hypothermic selective antegrade cerebral perfusion, using the right axillary artery for arterial inflow, with an open anastomosis technique. In the case of a dissected right axillary artery, we cannulated the femoral and 1 or both carotid arteries. The aortic arch remained unreplaced in patients with a nonaneurysmatic arch $(<5.0 \mathrm{~cm})$ and no intimal entry tear localized in the arch found on visual inspection and imaging. In patients undergoing ascending aorta replacement only, distal anastomosis was also performed using an open anastomotic technique. Hemiarch replacement was performed in patients with an aortic arch aneurysm $(>5.0 \mathrm{~cm})$ or intimal entry tear localized along the small curvature. In patients with an intimal entry tear localized along the greater curvature and those with a known connective tissue disorder, total arch replacement was performed. The dissected layers of the aorta were sealed with BioGlue (CryoLife Inc, Kennesaw, Ga).

\section{Patient Follow-up}

Surveillance follow-up data were obtained from the aortic clinic office visits and by interviewing the patients' primary care physician or the patients and their family members. Complete follow-up data were available for 127 of the 131 hospital survivors (97\%). The patients were followed up for a total of 733 patient-years, with a median follow-up among survivors of 4.9 years (first quartile, 2.5; third quartile, 7.6). Of the 131 survivors, $59(45 \%)$ were followed up for $\geq 5$ years.

In accordance with the current guidelines, ${ }^{1}$ the follow-up protocol included postoperative computed tomography angiography before discharge and clinical examination and computed tomography angiography at 6 and 12 months postoperatively and annually thereafter at our institutional aortic outpatient clinic or their regional hospitals for patients living in remote areas.

\section{Statistical Analysis}

Continuous data are presented as the median (first quartile; third quartile); categorical variables are given as counts and percentages. For comparison of continuous variables the Student $t$ test was applied when normal distribution was present as tested using the Kolmogorov-Smirnov test. For non-normally distributed variables, the Mann-Whitney rank sum test was used. Categorical variables were compared using the chi-square test. In the case of small group sizes $(\mathrm{n}<5)$, Fisher's exact test was used. The $P$ values were not presented for $\mathrm{n}=0$ in the subgroup. Survival was analyzed using the Kaplan-Meier method and log-rank calculations. Multivariable logistic regression was applied to analyze the influence of age $>80$ years, cardiogenic shock, malperfusion of $\geq 1$ organs, and hemi- or total arch replacement on in-hospital mortality and age $<45$ years, Marfan syndrome, dissection of all aortic segments, and incomplete resection of the dissected aortic arch tissue on the incidence of distal aortic reintervention among the survivors. All statistical calculations were performed using SigmaPlot, version 12 (Systat Software, San Jose, Calif).

\section{RESULTS \\ Surgical Treatment}

Aortic repair was limited to the ascending aorta in 102 patients $(67 \%)$, including 5 with unrecognized Marfan syndrome at surgery. Hemiarch replacement was performed in 37 patients $(24 \%)$ and total arch replacement in 14 patients $(9 \%)$. Also, 6 patients $(4 \%)$ underwent antegrade stent graft implantation into the descending thoracic aorta concomitant with hemiarch replacement. Of the 37 patients with dissection extending into the aortic arch but terminating proximal to the left subclavian artery $(24 \%), 15$ patients underwent resection of the entire dissected arch tissue and hemi- or total arch replacement was performed. In 22 patients, a lamellar dissection persisted within the arch.

Proximal aortic repair involved 105 aortic valve resuspensions, 37 aortic root replacements, 5 Wheat procedures (supracoronary ascending and aortic valve replacement), and 6 David operations.

\section{In-Hospital Mortality and Survival}

The lowest mortality was observed in patients with aortic replacement confined to the ascending aorta $(9.8 \%)$ and the greatest in those who underwent total arch replacement $(28.6 \% ; P=.122$; Table 1$)$. The total number of hospital deaths was 22 . The primary reason for in-hospital death was multisystem organ failure in $7(32 \%)$, heart failure in $5(23 \%)$, exsanguination due to postoperative bleeding in $4(18 \%)$, descending aortic rupture in $3(14 \%)$, and neurologic events in 3 patients $(14 \%)$. The overall survival was $88 \%$ (range, $80 \%-94 \%$ ), $81 \%$ (range, $63 \%-93 \%$ ), and $79 \%$ (range, $47 \%-92 \%$ ) at 1 year and $81 \%$ (range, $71 \%-88 \%$ ), 64\% (range, $41 \%-79 \%$ ), and $79 \%$ (range, $47 \%-92 \%)$ at 5 years in patients with isolated ascending aortic, hemi-, and total arch replacement, respectively (log-rank, $P=.062$; Figure 1).

\section{In-Hospital Mortality Risk Factors}

Using multivariable logistic regression analysis, age $>80$ years (odds ratio [OR], 9.37; $P=.006$ ), malperfusion of $\geq 1$ organs (OR, 4.74; $P=.004)$, and total arch replacement (OR, 6.47; $P=.016$ ) were identified as independent predictors of in-hospital mortality (Table 2).

\section{Distal Aortic Reintervention}

Freedom from distal reintervention was $93 \% \pm 3 \%$, $97 \% \pm 3 \%$, and $100 \%$ at 1 year and $89 \% \pm 3 \%, 97 \%$ $\pm 3 \%$, and $100 \%$ at 5 years in patients with isolated ascending aortic, hemi-, and total arch replacement, respectively (log-rank, $P=.440$; Figure 2 ). Twelve patients $(8 \%)$ required secondary procedures at a median of 1.0 year (first quartile, 0.4; third quartile, 1.8): 6, thoracic endovascular aortic repair; 3, hybrid arch debranching; 2 , total arch replacement; and 1, descending thoracic aortic 
TABLE 1. Demographic data, clinical presentation, and immediate outcomes

\begin{tabular}{|c|c|c|c|c|}
\hline Variable & No arch replacement $(n=102)$ & Hemiarch replacement $(n=37)$ & Total arch replacement $(n=14)$ & $P$ value \\
\hline Age (y) & $59(49 ; 70)$ & $66(59 ; 72)$ & $55(46 ; 63)$ & $.006 *$ \\
\hline Age $>80 y$ & $4(4)$ & $4(11)$ & $1(7)$ & .305 \\
\hline Male sex & $74(73)$ & $21(57)$ & $8(57)$ & .149 \\
\hline \multicolumn{5}{|l|}{ Clinical presentation } \\
\hline Hypertension & $75(74)$ & $31(84)$ & $13(93)$ & .159 \\
\hline Previous cardiac surgery & $2(2)$ & $1(3)$ & 0 & \\
\hline MFS & $5(5)$ & $2(5)$ & 0 & \\
\hline BAV & $4(4)$ & $3(8)$ & $1(7)$ & .584 \\
\hline Cardiogenic shock & $11(11)$ & $3(8)$ & $1(7)$ & .842 \\
\hline \multicolumn{5}{|l|}{ Malperfusion } \\
\hline$\geq 1$ Organs & $35(34)$ & $14(38)$ & $2(14)$ & .263 \\
\hline Coronary & $9(9)$ & $5(14)$ & 0 & \\
\hline Cerebral & $17(17)$ & $6(16)$ & $2(14)$ & .975 \\
\hline Iliofemoral & $10(10)$ & $5(14)$ & $2(14)$ & .441 \\
\hline Gastrointestinal & $7(7)$ & $3(8)$ & $1(7)$ & .969 \\
\hline \multicolumn{5}{|l|}{ Involvement of aortic dissection } \\
\hline Supra-aortic vessels & $58(57)$ & $17(46)$ & $3(21)$ & .035 \\
\hline Descending thoracic aorta & $80(78)$ & $27(73)$ & $9(64)$ & .459 \\
\hline Abdominal aorta & $64(63)$ & $21(57)$ & $7(50)$ & .587 \\
\hline Iliac vessels & $38(37)$ & $14(38)$ & $6(43)$ & .921 \\
\hline \multicolumn{5}{|l|}{ ECC details } \\
\hline ECC time (min) & $189(157 ; 233)$ & $196(163 ; 227)$ & $274(230 ; 351)$ & $<.001 \dagger$ \\
\hline Aortic crossclamp time (min) & $97(74 ; 139)$ & $109(80 ; 134)$ & $134(115 ; 177)$ & $<.001 \dagger$ \\
\hline ACP time (min) & $25(16 ; 36)$ & $32(19,41)$ & $71(46 ; 106)$ & $<.001 \dagger$ \\
\hline \multicolumn{5}{|l|}{ Outcome } \\
\hline In-hospital mortality & $10(10)$ & $8(22)$ & $4(29)$ & .122 \\
\hline Reoperation for bleeding & $7(7)$ & $3(8)$ & $2(14)$ & .624 \\
\hline Stroke, new onset & $9(9)$ & $2(5)$ & $1(7)$ & .868 \\
\hline TIA & $11(11)$ & $7(19)$ & $4(29)$ & .137 \\
\hline Respiratory failure & $5(5)$ & $3(8)$ & 0 & - \\
\hline
\end{tabular}

Data presented as median (first quartile; third quartile) for continuous values or n (\%) for categorical values. MFS, Marfan syndrome; $B A V$, bicuspid aortic valve; $E C C$, extracorporeal circulation; $A C P$, antegrade cerebral perfusion; $T I A$, transient ischemic attack. *Hemiarch versus total arch replacement. $\dagger$ No arch replacement versus total arch replacement.

replacement (Table 3). Reintervention was performed in 10 patients electively and in 2 emergently. Two patients who underwent emergency reoperation did not survive the secondary intervention. One died of multisystem organ failure after redo total arch replacement and one of ruptured descending thoracic aortic aneurysm 1 day after endovascular thoracic aortic repair. The in-hospital mortality for elective distal reintervention was $0 \%$.

\section{Distal Aortic Reintervention Risk Factors}

Marfan syndrome (OR, 12.40; $P=.038)$ and dissection of all aortic segments (OR, 10.68; $P=.007)$ were independent predictors of distal aortic reintervention. Of the 12 patients who underwent distal reintervention, aortic dissection involved all aortic segments in 11 patients. In 1 patient, the dissection primarily extended into the celiac trunk. Age $<45$ years $(\mathrm{OR}, 2.28 ; P=.379)$ and incomplete resection of the dissected aortic arch (OR, $0 ; P=.996)$ were not associated with aortic reintervention during the follow-up period.

\section{Perioperative Neurologic Complications}

Of the 153 patients with a dissected aortic arch, new-onset postoperative stroke occurred in 9 of 102

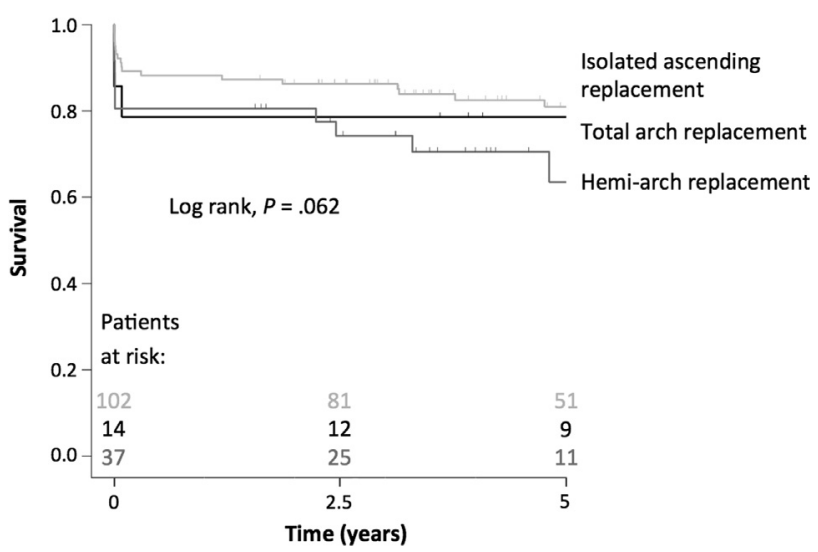

FIGURE 1. Kaplan-Meier analysis of overall survival of patients with dissected aortic arch who underwent isolated ascending, hemi-, or total arch replacement for acute type A aortic dissection. 
TABLE 2. In-hospital mortality risk factors for acute type A dissection surgical treatment in patients with dissected arch

\begin{tabular}{lllc}
\hline \multicolumn{1}{c}{ Variable } & OR & \multicolumn{1}{c}{$\mathbf{9 5} \% \mathbf{C I}$} & $\boldsymbol{P}$ value \\
\hline Age $>80 \mathrm{y}$ & 9.37 & $1.92-45.70$ & .006 \\
Cardiogenic shock & 3.40 & $0.79-14.53$ & .099 \\
Malperfusion in $\geq 1$ organs & 4.74 & $1.63-13.80$ & .004 \\
Hemiarch replacement & 2.43 & $0.77-7.63$ & .128 \\
Total arch replacement & 6.47 & $1.42-29.40$ & .016 \\
\hline$O R$, Odd
\end{tabular}

$O R$, Odds ratio; $C I$, confidence interval.

$(8.8 \%), 2$ of $37(5.4 \%)$, and 1 of $14(7.1 \%)$ with isolated ascending aortic, hemi-, and total arch replacement, respectively $(P=.868)$. The greatest transient ischemic attack incidence was observed in those who had undergone total arch replacement (4 of 14), but this difference did not reach statistical significance (10.8\% vs $18.9 \%$ vs $28.6 \%$, $P=.137)$.

\section{DISCUSSION}

In the setting of acute Stanford type A dissection, aortic dissection will infrequently be confined to only the ascending aorta. Concordant with other reports, ${ }^{4,8}$ dissection limited to the short proximal segment was observed in only every fifth patient in our study. In these patients, the entire dissected tissue can be replaced by isolated ascending aortic replacement. However, in most patients, the dissection process will extend beyond the ascending aorta, and extension of the distal repair to the aortic arch replacement will not necessarily eliminate the entire dissected tissue. It is still controversial whether the additional risk associated with aortic arch replacement is justified.

\section{Hospital Mortality and Neurologic Complications}

Despite vast improvements in diagnostic imaging and aortic surgery, the perioperative mortality rate for

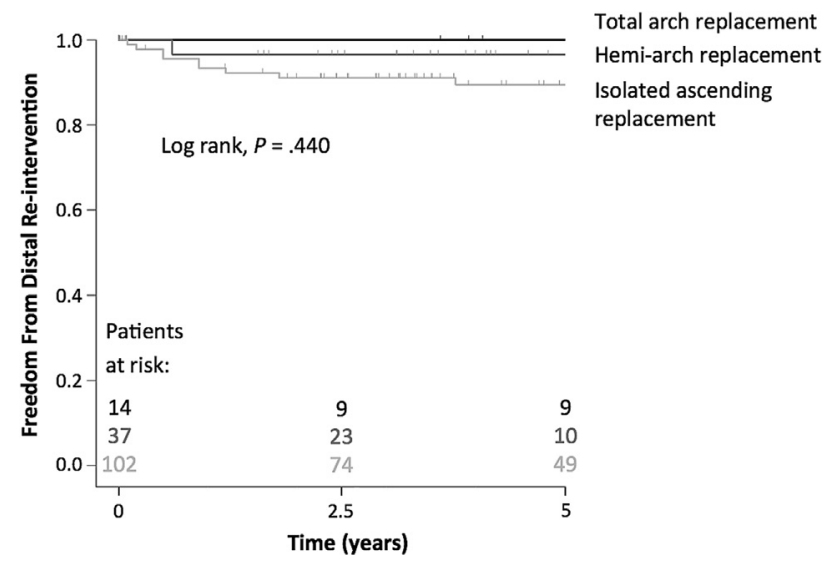

FIGURE 2. Kaplan-Meier analysis of freedom from distal aortic reintervention for patients with a dissected aortic arch who underwent isolated ascending, hemi-, or total arch replacement for acute type A aortic dissection. patients with acute type A dissection has remained at $8 \%$ to $34 \%{ }^{6,7,9} \mathrm{We}$ identified advanced age ( $>80$ years), malperfusion of $\geq 1$ organs, and total arch replacement as significant determinants of in-hospital mortality in patients with dissection extending beyond the ascending aorta. When stratified according to the initial aortic repair, the greatest mortality was observed in those with total arch replacement. Similar observations reported by others identified total arch replacement, not only as a perioperative mortality risk factor, ${ }^{10-13}$ but also as an independent risk factor for permanent neurologic injury. ${ }^{11}$

\section{Distal Aortic Reintervention}

A patent false lumen is the major and well-established risk factor for the need for reintervention on the aortic arch or descending aorta after type A dissection repair. ${ }^{14}$ Therefore, some groups have advocated a more aggressive approach for aortic arch repair to minimize the need for later reintervention. Tsagakis and colleagues ${ }^{2}$ published their experience with 44 patients with aortic dissection limited to the ascending aorta and aortic arch. They performed hemiarch repair in $37 \%$ and complete arch replacement in $36 \%$ of patients, with no need for distal aortic reintervention during a median follow-up of 2 years. The Philadelphia group ${ }^{15}$ has routinely performed hemiarch replacement in $>90 \%$ of all patients with type A dissection, with freedom from distal reoperation at 10 years of $76 \%$. Halstead and colleagues ${ }^{16}$ reported in a cohort of 179 patients, with $54 \%$ undergoing hemiarch and $6 \%$ total arch replacement, with a total of 25 reoperations within 17 years. Recently, several groups have reported even more aggressive approaches involving frozen elephant trunk implantation into the proximal descending thoracic aorta $^{17,18}$ or antegrade endovascular descending aortic repair $^{2,19}$; however, data on the long-term outcomes are lacking.

In the present study, aortic arch replacement was performed to eliminate the intimal tear or replace an aneurysmatic aortic arch. To minimize the risk of cerebral injuries and organ dysfunction related to the longer duration of cardiac ischemia and circulatory arrest, our group does not routinely perform prophylactic arch replacement. In our series of patients with a dissected aortic arch, the dissection extended beyond the arch in $76 \%$ of the patients. Therefore, routine arch replacement would not have eliminated the entire dissected tissue in most of our patients. For 37 patients with dissection terminating at the left subclavian artery, we chose a more conservative surgical strategy that was limited to isolated ascending aortic replacement in 22 patients, because resection of all the dissected tissue in those patients would have required total aortic arch replacement. We performed total aortic arch replacement only if it was unavoidable owing to an arch aneurysm or intimal tear along the greater curvature. 
TABLE 3. Distal aortic reintervention

\begin{tabular}{|c|c|c|c|c|c|}
\hline Pt. no. & Year & Initial operation & $\begin{array}{l}\text { Indication for } \\
\text { reintervention }\end{array}$ & Re-intervention & Interval $(\mathbf{y})$ \\
\hline 1 & 2005 & No arch replacement & TAA & TEVAR & 0.5 \\
\hline 2 & 2007 & No arch replacement & Arch aneurysm & Hybrid arch debranching* & 0.1 \\
\hline 3 & 2008 & No arch replacement & Arch aneurysm & Hybrid arch debranching $\dagger$ & 1.0 \\
\hline 4 & 2008 & No arch replacement & TAA & TEVAR & 3.8 \\
\hline 5 & 2009 & No arch replacement & TAA & TEVAR & 0.2 \\
\hline 6 & 2009 & No arch replacement & TAA & TEVAR & 0.9 \\
\hline 7 & 2009 & No arch replacement & TAA & TEVAR & 0.5 \\
\hline 8 & 2009 & Hemiarch replacement & Arch aneurysm & Redo total arch replacement & 7.3 \\
\hline 9 & 2010 & No arch replacement & TAA & TAA repair & 1.8 \\
\hline 10 & 2010 & No arch replacement & Arch aneurysm & Redo total arch replacement & 8.8 \\
\hline 11 & 2010 & Hemiarch replacement & TAA & TEVAR & 0.1 \\
\hline 12 & 2012 & No arch replacement & Arch aneurysm & Hybrid arch debranching* & 1.2 \\
\hline
\end{tabular}

Pt. no., Patient number; TAA, thoracic aortic aneurysm; TEVAR, thoracic endovascular aortic repair. *Left carotid-subclavian bypass implantation and endovascular aortic arch repair with stent deployment beginning in zone 0 distal to the aortic bicarotid bypass performed during the initial procedure. $\dagger$ Redo sternotomy, establishment of brachiocephalic and left carotid bypass (both proximally anastomosed to the ascending graft), and endovascular aortic arch repair.

Marfan syndrome and dissection of all aortic segments were predictors of distal aortic reintervention. Incomplete resection of the dissected aortic arch did not correlate with the need for distal reintervention. Among the patients who underwent distal reoperation, the dissection had extended to the celiac trunk in $100 \%$ and to the iliac arteries in $92 \%$ at the initial presentation. These data highlight the finding that extending the initial surgery to total arch replacement would not have eliminated the entire dissected tissue in any of these patients. However, none of the patients who had initially undergone total arch replacement required reintervention during the follow-up period, and none of the patients surviving type A dissection surgery had died within 5 years after hospital discharge ( 9 patients remaining at risk). The disadvantage of this favorable outcome is the high risk of perioperative mortality associated with the initial surgery. It is conceivable that younger patients could benefit from more aggressive surgery. However, future studies with longer follow-up periods are needed. In particular, the frozen elephant technique might be a favorable option for the treatment of young patients or patients with connective tissue disorders to establish the best possible replacement of dissected tissue and optimize long-term survival.

Elective reintervention was performed safely with no observed perioperative mortality. These data suggest that limiting the surgery for type A dissection to ascending aortic replacement will result in low perioperative mortality $(9.8 \%)$ and that distal aortic reintervention can be performed safely during the follow-up period, if secondary aortic pathologic features are diagnosed in time to allow for elective procedures. A shorter interval ( $<6$ months) between discharge and the first follow-up imaging study should be considered to avoid emergency reintervention, especially in patients at higher risk of reintervention such as those with connective tissue disorder and extensive aortic dissection pathologic features.

\section{Study Limitations}

The present study was limited by several factors. First, this was a retrospective, observational study with all of the inherent drawbacks. The extension of aortic arch surgery was not chosen at random but was influenced by the presence of arch aneurysm, intimal tear localization, and known connective tissue disorder. Third, we found no association between young age at dissection and distal aortic reoperation. However, given the median follow-up period of 5 years, larger studies with longer follow-up data could provide more insights into this field. Finally, the risk factors for in-hospital mortality and distal reintervention should be considered with caution owing to the wide confidence intervals, as expected given the sample size.

\section{CONCLUSIONS}

Considering the advances in aortic surgery during the past decade, complex aortic arch surgery in the setting of acute type A aortic dissection is still associated with high perioperative mortality. In patients with dissection extending beyond the ascending aorta, limiting emergency surgery to life-preserving procedures, such as ascending arch replacement, aortic valve insufficiency correction, and restoring flow to the dissected branch vessels, has been associated with low perioperative mortality. Deferring additional aortic arch procedures might be justified, because they can be performed electively with a lower risk of perioperative mortality.

\section{References}

1. Hiratzka LF, Bakris GL, Beckman JA, Bersin RM, Carr VF, Casey DE, et al. 2010 ACCF/AHA/AATS/ACR/ASA/SCA/SCAI/SIR/STS/SVM guidelines for the diagnosis and management of patients with thoracic aortic disease: a report of the American College of Cardiology Foundation/American Heart Association Task Force on Practice Guidelines, American Association for Thoracic Surgery, American College of Radiology, American Stroke Association, Society of 
Cardiovascular Anesthesiologists, Society for Cardiovascular Angiography and Interventions, Society of Interventional Radiology, Society of Thoracic Surgeons, and Society for Vascular Medicine. Circulation. 2010;121:e266-369.

2. Tsagakis K, Tossios P, Kamler M, Benedik J, Natour D, Eggebrecht H, et al The DeBakey classification exactly reflects late outcome and re-intervention probability in acute aortic dissection with a slightly modified type II definition. Eur J Cardiothorac Surg. 2011;40:1078-84.

3. DeBakey ME, Henly WS, Cooley DA, Morris GC Jr, Crawford ES, Beall AC Jr. Surgical management of dissecting aneurysms of the aorta. J Thorac Cardiovasc Surg. 1965;49:130-49.

4. Rylski B, Hoffmann I, Beyersdorf F, Suedkamp M, Siepe M, Nitsch B, et al. Acute aortic dissection type A: age-related management and outcomes reported in the German Registry for Acute Aortic Dissection Type A (GERAADA) of over 2000 patients. Ann Surg. 2014;259:598-604.

5. Rylski B, Beyersdorf F, Blanke P, Boos A, Hoffmann I, Dashkevich A, et al. Supracoronary ascending aortic replacement in patients with acute aortic dissection type A: what happens to the aortic root in the long run? J Thorac Cardiovasc Surg. 2013; 146:285-90.

6. Rylski B, Suedkamp M, Beyersdorf F, Nitsch B, Hoffmann I, Blettner M, et al. Outcome after surgery for acute aortic dissection type A in patients over 70 years: data analysis from the German Registry for Acute Aortic Dissection Type A (GERAADA). Eur J Cardiothorac Surg. 2011;40:435-40.

7. Dell'aquila AM, Concistrè G, Gallo A, Pansini S, Piccardo A, Passerone G, et al. Fate of the preserved aortic root after treatment of acute type A aortic dissection: 23-year follow-up. J Thorac Cardiovasc Surg. 2013;146:1456-60.

8. Urbanski PP, Siebel A, Zacher M, Hacker RW. Is extended aortic replacement in acute type A dissection justifiable? Ann Thorac Surg. 2003;75:525-9.

9. Rylski B, Hoffmann I, Beyersdorf F, Suedkamp M, Siepe M, Nitsch B, et al. Iatrogenic acute aortic dissection type A: insight from the German Registry for Acute Aortic Dissection Type A (GERAADA), Eur J Cardiothorac Surg. 2013;44:353-9.

10. Kim JB, Chung CH, Moon DH, Ha GJ, Lee TY, Jung SH, et al. Total arch repair versus hemiarch repair in the management of acute DeBakey type I aortic dissection. Eur J Cardiothorac Surg. 2011;40:881-7.

11. Pagni S, Ganzel BL, Trivedi JR, Singh R, Mascio CE, Austin EH, et al. Early and midterm outcomes following surgery for acute type A aortic dissection. J Card Surg. 2013;28:543-9.

12. Westaby S, Saito S, Katsumata T. Acute type A dissection: conservative methods provide consistently low mortality. Ann Thorac Surg. 2002;73:707-13.

13. Ohtsubo S, Itoh T, Takarabe K, Rikitake K, Furukawa K, Suda H, et al. Surgical results of hemiarch replacement for acute type A dissection. Ann Thorac Surg. 2002; 74:S1853-6.

14. Concistre G, Casali G, Santaniello E, Montalto A, Fiorani B, Dell'Aquila A, et al. Reoperation after surgical correction of acute type A aortic dissection: risk factor analysis. Ann Thorac Surg. 2012;93:450-5.

15. Geirsson A, Bavaria JE, Swarr D, Keane MG, Woo YJ, Szeto WY, et al. Fate of the residual distal and proximal aorta after acute type A dissection repair using a contemporary surgical reconstruction algorithm. Ann Thorac Surg. 2007;84:1955-64.

16. Halstead JC, Meier M, Etz C, Spielvogel D, Bodian C, Wurm M, et al. The fate of the distal aorta after repair of acute type A aortic dissection. J Thorac Cardiovasc Surg. 2007; 133:127-35.

17. Sun LZ, Qi RD, Chang Q, Zhu JM, Liu YM, Yu CT, et al. Surgery for acute type A dissection using total arch replacement combined with stented elephant trunk implantation: experience with 107 patients. J Thorac Cardiovasc Surg. 2009;138:1358-62.

18. Uchida N, Shibamura H, Katayama A, Shimada N, Sutoh M, Ishihara H. Operative strategy for acute type A aortic dissection: ascending aortic or hemiarch versus total arch replacement with frozen elephant trunk. Ann Thorac Surg. 2009;87:773-7.

19. Pochettino A, Brinkman WT, Moeller P, Szeto WY, Moser W, Cornelius K, et al. Antegrade thoracic stent grafting during repair of acute DeBakey I dissection prevents development of thoracoabdominal aortic aneurysms. Ann Thorac Surg. 2009;88:482-9.

\section{Discussion}

Dr Leonard N. Girardi (New York, NY). Thank you very much and great presentation. I have 3 questions for you.

It seems although you could not prove that hemiarch replacement was any different from no arch to total arch statistically, when you look at the raw data, the mortality risk for a hemiarch was $21.5 \%$ versus $9.8 \%$ for your no arch replacement.

Also, when you study the report and the technique for how you do it, you are still using circulatory arrest. You are still using antegrade perfusion. The postoperative stroke risks are the same, so I am not following where the difference in mortality is that would justify just doing an open repair and limiting it to the ascending aorta. So, is there something I am missing in the data?

Dr Siepe. Well, actually, that affects the reasons for death. We could not find any statistical difference for the reasons for death in the different groups. But the most common reason was multiorgan failure, likely due to the more extensive operation in the total arch group.

One confounding factor could be that different types of patients are treated in different ways. We could not find all the confounding factors, in particular, the intraoperative decision of the surgeon in the specific situation is difficult to put in a retrospective objective analysis.

Dr Girardi. Well, the antegrade perfusion time, however, between the no arch and the hemiarch was only 7 minutes. For the pump times, there was a minimal difference in that as well. So the technique itself does not seem to be the reason that the patients had multiple organ failure, and I am still not clear as to why that is.

My second question concerns the patients who presented with cerebral malperfusion, which constituted about $15 \%$ to $17 \%$ of all 3 groups. Again, although the stroke rate was statistically not different among the groups, the stroke rate in the no arch group was $9 \%$ versus $4 \%$ or $5 \%$ for the other groups.

How did you know in the patients with cerebral malperfusion that just doing an ascending was enough? If you go out into the arch, you could find something that you can correct that might make it a little bit better, so how were you able to sort that out before you decided what to do?

Dr Siepe. Well, I think the answer to your excellent question is not very clear from this retrospective point. Also, after total arch replacement and involvement of the carotid arteries in the dissection process, you cannot be sure that you have adequate blood flow through to the brain. That is why we routinely use monitoring tools such as near-infrared spectroscopy measurement. That is actually the most sensitive method for detecting that there might be a problem, but that is a weak indication I confess.

Dr Girardi. Finally, if you consider the reinterventions, the median interval to reintervention was 1 year, and 10 of the 12 patients who underwent reintervention did so at around 1 year and even earlier, some as soon as 2 months postoperatively. Also, if you remove the 2 patients who underwent reintervention at 7 and 8 years, the median was probably 9 months. It seems relatively early for reintervention, and can you just comment on what was the reason for such early reintervention in these patients? Thank you.

Dr Siepe. Thank you for this excellent question. The reason for this early reintervention was mainly that on the follow-up computed tomography scan, significant disease of the downstream aorta was highlighted. It was mainly the descending aorta.

In our routine work, we do not only treat the descending aorta after dissection if the diameter is too large, but also if the true lumen is very small. Thus, perhaps we can establish a better true lumen and close the false lumen in the descending aorta. Some patients were treated like that, which might account for the short interval between the operation and the intervention. 